\title{
Dosimetry for spectral molecular imaging of small animals with MARS-CT
}

\author{
Noémie Ganet ${ }^{\mathrm{a}, \mathrm{d}}$, Nigel Anderson ${ }^{\mathrm{b}}$, Stephen Bell ${ }^{\mathrm{c}}$, Anthony Butler ${ }^{\mathrm{a}, \mathrm{b}, \mathrm{c}, \mathrm{d}}$, Phil Butler ${ }^{\mathrm{a}, \mathrm{b}, \mathrm{c}, \mathrm{d}}$, Pierre \\ Carbonez $^{\mathrm{a}}$, Nicholas Cook*e, Tony Cotterill ${ }^{\mathrm{f}}$, Steven Marsh ${ }^{\mathrm{d}}$, Raj Kumar Panta ${ }^{\mathrm{b}}$, John Laban ${ }^{\mathrm{f}}$, Sophie \\ Walker ${ }^{\mathrm{f}}$, Adam Yeabsley ${ }^{\mathrm{f}}$, Jérôme Damet ${ }^{\mathrm{a}, \mathrm{g}}$ \\ aCERN, CH-1211 Geneva 23, Switzerland, bDept. of Radiology, University of Otago, Christchurch, \\ New Zealand, 'MARS Bioimaging Ltd, Christchurch, New Zealand, dUniversity of Canterbury, \\ Christchurch, New Zealand, ${ }^{e}$ Medical Physics and Bio-Engineering, Canterbury District Health

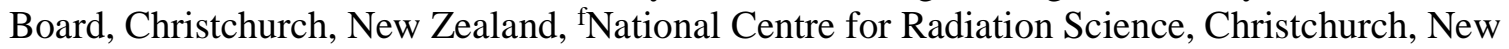 \\ Zealand, 'Institute of Radiation Physics, Lausanne University Hospital, Lausanne, Switzerland
}

\begin{abstract}
The Medipix All Resolution Scanner (MARS) spectral CT is intended for small animal, pre-clinical imaging and uses an $\mathrm{x}$-ray detector (Medipix) operating in single photon counting mode. The MARS system provides spectrometric information to facilitate differentiation of tissue types and bio-markers. For longitudinal studies of disease models, it is desirable to characterise the system's dosimetry. This dosimetry study is performed using three phantoms each consisting of a $30 \mathrm{~mm}$ diameter homogeneous PMMA cylinder simulating a mouse. The imaging parameters used for this study are derived from those used for gold nanoparticle identification in mouse kidneys. Dosimetry measurement are obtained with thermo-luminescent Lithium Fluoride ( $\mathrm{LiF}: \mathrm{CuMgP}$ ) detectors, calibrated in terms of air kerma and placed at different depths and orientations in the phantoms. Central axis TLD air kerma rates of $17.2( \pm 0.71) \mathrm{mGy} / \mathrm{min}$ and $18.2( \pm 0.75)$ $\mathrm{mGy} / \mathrm{min}$ were obtained for different phantoms and TLD orientations. Validation measurements were acquired with a pencil ionization chamber, giving an air-kerma rate of $20.3( \pm 1) \mathrm{mGy} / \mathrm{min}$ and an estimated total air kerma of $81.2( \pm 4)$ $\mathrm{mGy}$ for a 720 projection acquisition. It is anticipated that scanner design improvements will significantly decrease future dose requirements. The procedures developed in this work will be used for further dosimetry calculations when optimizing image acquisition for the MARS system as it undergoes development towards human clinical applications.
\end{abstract}

Keywords: dosimetry, computed tomography, thermoluminescent, molecular imaging, spectral imaging, medipix, longitudinal, small-animal

\section{INTRODUCTION}

Spectral $\mathrm{CT}^{1}$ is a developing technology which is generating interest as a potential next step in pre-clinical and eventually human imaging. By accurately characterizing the spectral attenuation of X-rays for each voxel, new information is available that may improve diagnosis and therapy monitoring. Potentially, spectral CT can simultaneously differentiate multiple tissue components, contrast media, and high Z (atomic number) nanoparticle bio-markers ${ }^{2}$. Significant challenges remain before spectral CT is available for human scanning, including: detector design, novel reconstructions ${ }^{3}$, photon counting rates ${ }^{4}$, and dose optimisation ${ }^{5}$.

The Centre for Bioengineering at the University of Otago, together with national and international partners has developed a research spectral CT scanner for small animal studies. This MARS (Medipix All Resolution Scanner) scanner $^{6}$ uses the Medipix $3 \mathrm{RX}^{8}$ chip with high $\mathrm{Z}$ detector layers such as CZT (Cadmium Zinc Telluride) and CdTe to acquire spectral data sets. These data sets are reconstructed using an algebraic technique, analysed using constrained material decomposition, and visualised with specially developed in-house software ${ }^{6}$. The MARS scanner is intended for use in longitudinal small-animal studies. It is important to measure and analyze dose as the technology and applications develop, both to optimize scanner design and also to assess radiation risk and potential effects on longitudinal studies. This paper describes initial thermo-luminescent dosimetry (TLD) work with a comparison to pencil ionization chamber measurements.

*nick.cook@cdhb.health.nz; phone +64 3364 1868; fax +64 3364 1177; www.cdhb.health.nz 


\section{DOSIMETRY}

\subsection{TLD Dosimtery}

The TLDs used were of type GR-200A 9 , produced by the China Quartz Technology Group (Hangzhou, P. R. China). The TLDs are Lithium Fluoride ( $\mathrm{LiF}$ ) doped with $\mathrm{Cu}, \mathrm{Mg}$, and $\mathrm{P}$, they are cylindrical with diameter $4.5 \mathrm{~mm}$ and thickness $0.8 \mathrm{~mm}$. This type of TLD has certain advantages over the traditional $\mathrm{Li}: \mathrm{Mg}, \mathrm{Ti}$ including: higher sensitivity, improved energy response, lack of supralinearity, and insignificant fading. Based on a calculated average beam energy of 56keV, TLD output was expected to be within a few $\%$ of that for the Co-60 source ${ }^{10}$. A Harshaw model $5500^{11}$ automatic TLD reader (Thermo Scientific, Waltham, MA) was used to measure TLD output: measuring total charge by integrating the total area under the TLD glow peak. The manufacturer's recommended TLD heating profile was used as follows: preheating to $140^{\circ} \mathrm{C}$ over 10 seconds, followed by the readout phase at $10^{\circ} \mathrm{C} / \mathrm{s}$ increasing to $220^{\circ} \mathrm{C}$, then hold at $220^{\circ} \mathrm{C}$ for 20 seconds. TLDs were annealed for reuse by heating to $225^{\circ} \mathrm{C}$ for 30 minutes. TLD response had previously been confirmed to be linear to dose in the range $10 \mathrm{pGy}$ to $10 \mathrm{~Gy}$.

A population of 50 TLDs was used for the present work. Sensitivities of the individual TLDs relative to the mean response of the 50 was assessed by exposing the TLDs to a uniform beam of Co-60 radiation with an entrance air kerma of $50 \mathrm{mGy}$, and the response of each recorded. This was repeated 5 times, and the average relative response of each of the TLDs was calculated. The standard deviation (SD) of these 5 measurements was also calculated and two TLDs with $\mathrm{SD}>3 \%$ were removed from the batch, the average SD was $1.4 \%$ which gives an estimate of the uncertainty of the TLD reading process. All TLD readings obtained subsequently were normalised according to their respective relative sensitivities.

TLD response is energy dependent, though variations have been found to be relatively small ${ }^{10}$. Beam quality of the scanner is defined by: $120 \mathrm{kVp}, 20^{\circ}$ anode angle, inherent filtration $1.8 \mathrm{~mm} \mathrm{Al}$, added filtration $2 \mathrm{~mm} \mathrm{Al}$, Half Value Layer (HVL) $4.82 \mathrm{~mm} \mathrm{Al} \mathrm{(SpekCalc}{ }^{12,13}$ calculation). To confirm TLD response for this beam quality, groups of 8 TLDs were exposed to a known air-Kerma from 3 beams with qualities that bracketed the estimated scanner beam quality. The setup for beam quality calibrations is shown in Figure 1 , the TLDs were held face normal $\left(90^{\circ}\right)$ and face orthogonal $\left(0^{\circ}\right)$ to the beam axis using thin plastic food wrap. The 3 test beam qualities are shown in Table 1 with the scanner beam quality and the average of the 8 TLD readings for each case.

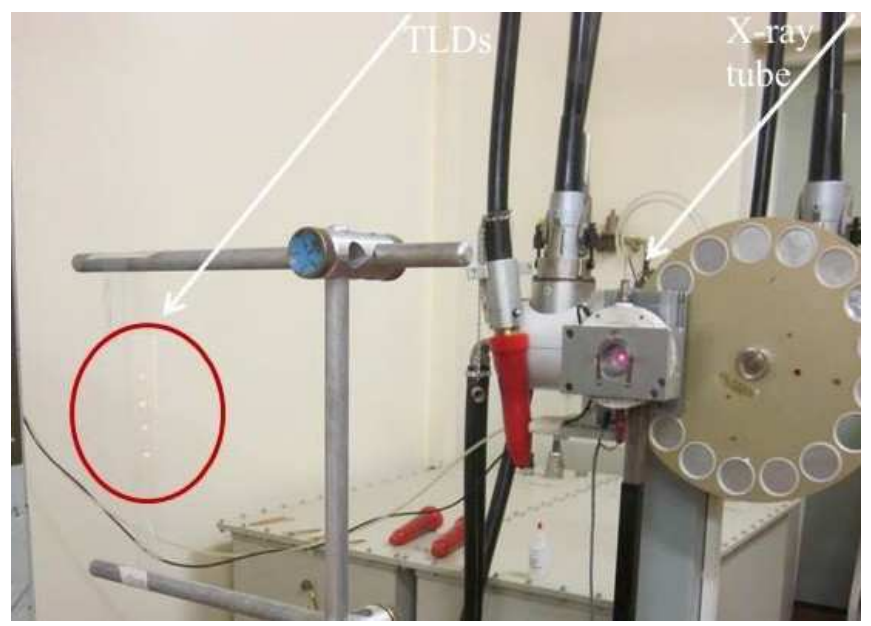

Figure 1. Photograph of the setup for beam quality and TLD orientation measurements. TLDs are held in position using thin plastic food wrap. 
Table 1 Beam qualities used to calibrate TLD energy response

\begin{tabular}{|l|l|l|l|l|l|}
\hline & \multicolumn{1}{|c|}{$\begin{array}{c}\text { Beam 1 } \\
(\mathbf{9 0})\end{array}$} & $\begin{array}{c}\text { Beam 1 } \\
\left(\mathbf{0}^{\circ}\right)\end{array}$ & $\begin{array}{c}\text { Beam 2 } \\
\left(\mathbf{0}^{\circ}\right)\end{array}$ & $\begin{array}{c}\text { Beam 3 } \\
\left(\mathbf{0}^{\circ}\right)\end{array}$ & $\begin{array}{c}\text { Scanner } \\
\mathbf{B e a m}^{\circ}\end{array}$ \\
\hline $\mathrm{kVp}$ & 120 & 120 & 120 & 120 & 120 \\
\hline Total filtration & $\begin{array}{l}3 \mathrm{~mm} \mathrm{Be}+ \\
3 \mathrm{~mm} \mathrm{Al}\end{array}$ & $\begin{array}{l}3 \mathrm{~mm} \mathrm{Be}+ \\
3 \mathrm{~mm} \mathrm{Al}\end{array}$ & $\begin{array}{l}3 \mathrm{~mm} \mathrm{Be}+ \\
4 \mathrm{~mm} \mathrm{Al}\end{array}$ & $\begin{array}{l}3 \mathrm{~mm} \mathrm{Be}+ \\
5 \mathrm{~mm} \mathrm{Al}\end{array}$ & $3.8 \mathrm{~mm} \mathrm{Al}$ \\
\hline HVL & $4.26 \mathrm{~mm} \mathrm{Al}$ & $4.26 \mathrm{~mm} \mathrm{Al}$ & $5.01 \mathrm{~mm} \mathrm{Al}$ & $5.63 \mathrm{~mm} \mathrm{Al}$ & $4.82 \mathrm{~mm} \mathrm{Al}$ \\
\hline Mean (nC/mGy) & 171.5 & 161.9 & 163.1 & 160.6 & \\
\hline Standard Deviation (nC/mGy) & 3.2 & 4.7 & 3.4 & 4.5 & \\
\cline { 1 - 3 } & &
\end{tabular}

To correct for possible TLD reader output variation between the time of calibration and CT scanner exposures, a subset of 8 TLDs was irradiated with Co-60 to $50 \mathrm{mGy}$ entrance air kerma at calibration and read out; these 8 TLDs were exposed and read again at the same time the CT scanner exposures were performed and read. Results from the CT scanner exposures were normalised according to the ratio of the results of the Co- 60 exposures. The average responses of the 8 TLDs to the Co-60 air-Kerma of 50mGy on calibration day and on reading day are shown in Table 2.

Table 2. Responses of 8 reference TLDs to Co-60 on calibration day and reading days

\begin{tabular}{|l|l|l|}
\hline & Calibration Day (nC/mGy) & \multicolumn{1}{|c|}{ Reading Day (nC/mGy) } \\
\hline Mean of 8 TLDs & 200.9 & 195.8 \\
\hline Standard Deviation & 1.58 & 3.6 \\
\hline
\end{tabular}

\subsection{Phantoms and TLD orientation}

During the beam quality tests, it was found that the orientation of TLDs could change measured dose by approximately 6\%. To estimate the dose response in small animals, two phantoms were therefore constructed from 30mm diameter PolyMethylMethAcrylate (PMMA) cylinder, one holding TLDs normal and the other orthogonal to the phantom surface. The TLDs were positioned in wells of various depth and each well was then filled with a PMMA plug, these phantoms are shown in Figure 2.
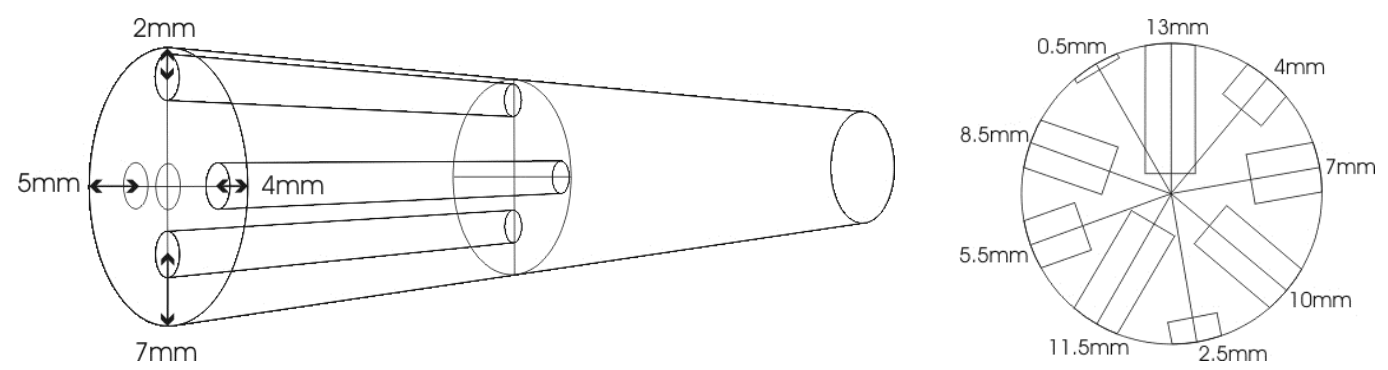

Figure 2 PMMA Phantoms: diameter 30mm, TLDs placed in various depth wells, filled with PMMA plugs.

These phantoms were positioned at the isocentre of the scanner with the TLDs in the centre of the beam profile and a typical mouse scanning protocol was used to assess dose.

\subsection{Pencil ionization chamber dosimetry}

To validate the TLD measurements, a third phantom was constructed from a 30mm diameter PMMA cylinder with a central hole to accept an Unfors Xi carbon fibre CT pencil chamber ${ }^{14}$ (Unfors RaySafe, Billdal, Sweden) with a 100mm long and $7.5 \mathrm{~mm}$ diameter active volume. It is therefore only possible to sample dose along the central axis of this phantom, however this allows comparison with and validation of centrally placed TLDs. The pencil chamber is automatically corrected for pressure and temperature variations and has an uncertainty of 5\% at reference point RQT9. 
The PMMA phantom with pencil chamber inserted is shown at the scanner isocentre in Figure 3 . The same scanning protocol was used to measure dose as described for TLD measurements below.

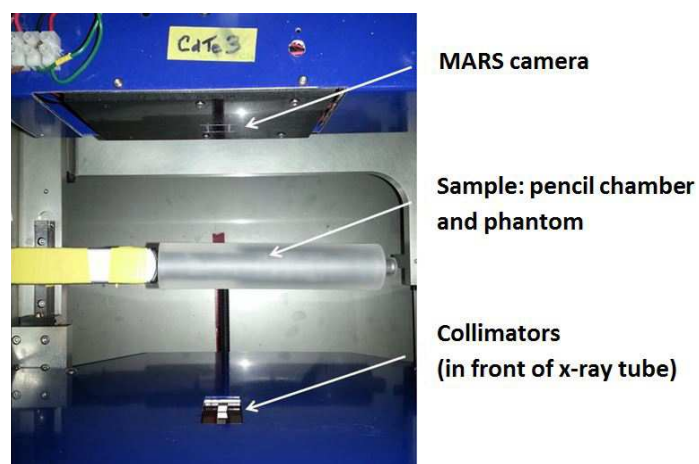

Figure 3. Pencil ionization chamber inserted in 30mm PMMA cylinder and placed at the scanner isocentre.

\section{MARS SCANNER}

The MARS (photographs in Figure 4) is a micro-CT scanner with a spectral Medipix3RX detector. It is designed to image small animals and samples with a possible length of $200 \mathrm{~mm}$ and diameter $80 \mathrm{~mm}$. The X-ray tube and detector assembly are mounted on screw drives to allow variable magnification and the enclosure is shielded to reduce radiation close to background levels, allowing scanning in unshielded rooms.
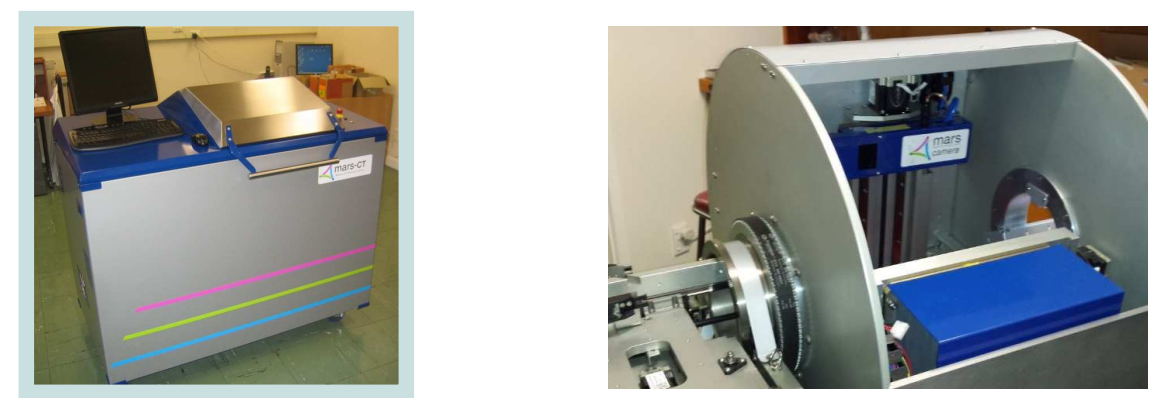

Figure 4. Photographs of the MARS spectral CT scanner and gantry, showing camera and X-ray assembly.

The Xray assembly is a Source-Ray SB-120-350 (Source-Ray Inc, Fleetwood, CT), using Superior X-ray tube SXR-13020-05 (Superior X-ray Tube Co., Woodstock, IL) with the following specifications: $70 \mu \mathrm{m}$ focal spot, 60 to $120 \mathrm{kVp}, 10$ to $350 \mu \mathrm{A}$, anode angle $20^{\circ}$, and inherent filtration $1.8 \mathrm{~mm} \mathrm{Al}$ at $120 \mathrm{kVp}$. For the measurements here, the scanner was configured with the following geometry: Source Object Distance (SOD) 140mm and Object Detector Distance (ODD) $80 \mathrm{~mm}$, giving a magnification factor of 1.57 .

The scan protocol followed for this work was adapted from a typical small animal study and used the following parameters: beam width $9 \mathrm{~mm}$, voltage $120 \mathrm{kVp}$, current $50 \mu \mathrm{A}$, and exposure time $90 \mathrm{~ms}$. Usually 720 projections would be acquired over $360^{\circ}$ but this was reduced to 180 projections to save time. Due to the development status of the scanner and camera, it was necessary to step the single Medipix detector chip 3 times to cover each projection field, the beam was left on during these steps.

\subsection{Medipix detector}

Medipix $3 \mathrm{RX}^{5}$ is a pixelated, hybrid, photon-counting detector that has up to 8 tunable energy threshold levels depending on the mode of operation. Accurate energy thresholding is possible thanks to a pixel-by-pixel equalization process and novel, on-chip charge-sharing correction methods. Using these techniques it is possible to generate spectral data with an energy resolution of a few $\mathrm{keV}$, much better than the energy resolution available from dual energy scanners with overlapping energy spectra or double-layer detector systems. In this work, the Medipix detectors utilized a CdTe detector 
layer; alternative detector materials such as CZT, GaAs, and silicon can also be bump-bonded to the same Medipix ASIC (Application Specific Integrated Circuit). Figure 5 shows photographs of the Medipix 3RX fingerboards which are mounted in a MARS camera module.
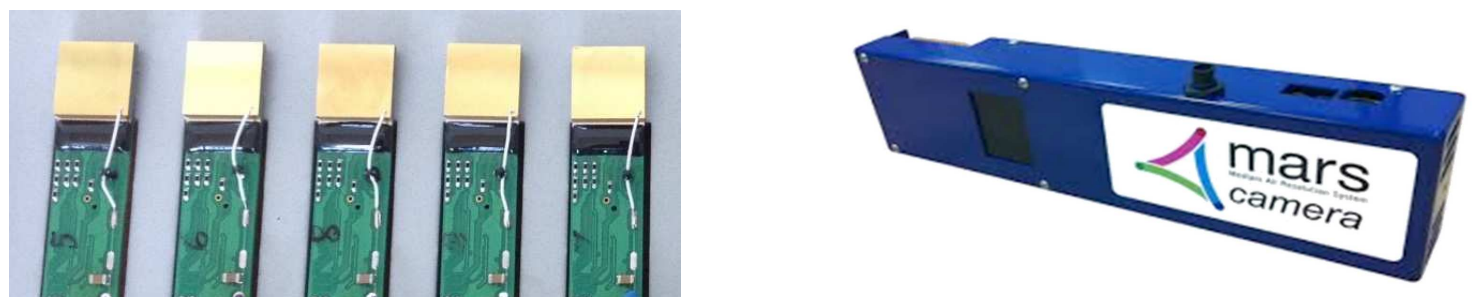

Figure 5. Medipix $3 R X$ fingerboards (left) which can be mounted in a $3 \times 2$ array in the MARS camera (right)

\section{RESULTS}

\subsection{TLD Results}

The TLDs were normalised and calibrated using the process described in section 2.1. Variations in TLD output over the range of tested beam qualities (HVL: 4.26 to $5.63 \mathrm{~mm} \mathrm{Al}$ ) were found to be of the same order as the standard deviation of the 8 TLDs used for each beam measurement, ie the TLDs were taken to have a flat energy response around the region of the CT scanner beam quality. Data from beam 2 was chosen as the basis for the energy response calibration of the TLDs. TLD orientation did make a significant difference (approximately $6 \%$ higher response for the $90^{\circ}$ orientation). This is likely due to self-attenuation in the TLD during the free-in-air calibration process. Results calculated from irradiations involving all orientations in a single exposure were corrected for by weighting the calibration factors used between the $0^{\circ}$ and $90^{\circ}$ extremes.

The variation of TLD air kerma rate with depth is shown for two tests using the two phantoms in Figure 6. The graph on the left shows results for TLDs oriented edge-on to the circumference of the phantom and the graph on the right is for TLDs oriented face-on to the circumference
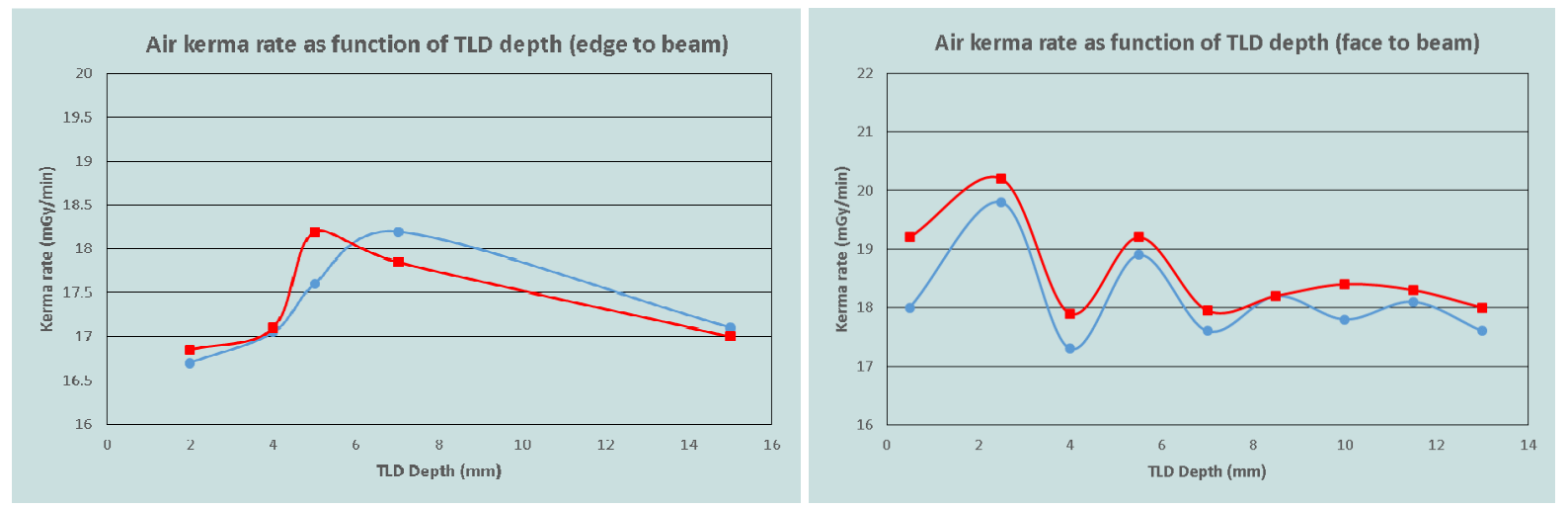

Figure 6. Air-kerma rate at various depths in PMMA phantoms. Left: TLD's oriented edge-on to phantom circumference. Right: TLD's oriented face-on to phantom circumference.

The most obvious feature of these results is that the air-kerma rate does not fall monotonically with depth as expected. This is due to a beam-on time of several seconds before gantry rotation and acquisitions start. The TLDs giving higher dose rates were positioned on the X-ray tube side of the phantom during this stationary time and received a higher than expected dose, unfortunately this means our depth-dose data is unreliable. The dose rates at the centre of the TLD phantoms, and the dose rate recorded by the pencil ionization chamber are shown in Table 3.Uncertainties are from 
published specifications for the pencil chamber and calculated from stated standard deviations for the various TLD calibration processes.

Table 3. Measured average dose rates close to centre of 30mm PMMA phantom. Centre depth is shortest distance from phantom surface to centre of detector, cross-section is the radial distance occupied by the detector volume.

\begin{tabular}{|l|l|l|l|l|}
\hline & $\begin{array}{l}\text { Centre Depth } \\
(\mathbf{m m})\end{array}$ & $\begin{array}{l}\text { Cross section } \\
(\mathbf{m m})\end{array}$ & $\begin{array}{l}\text { Dose rate } \\
(\mathbf{m G y} / \mathbf{m i n})\end{array}$ & $\begin{array}{l}\text { Estimated uncertainty } \\
\mathbf{\%}(\mathbf{m G y})\end{array}$ \\
\hline Pencil chamber & 15 & 7.5 & 20.3 & $5(1.02)$ \\
\hline TLD edge-on & 15 & 4.5 & 17.2 & $4.1(0.71)$ \\
\hline TLD face-on & 12.6 & 0.8 & 18.2 & $4.1(0.75)$ \\
\hline
\end{tabular}

The results in Table 3 show that the three methods of measuring dose rate at the centre of the phantom give similar results. The pencil chamber gives the highest value, perhaps because it has the largest cross section and phantom wall thickness is therefore reduced. The two TLD positions are measuring dose rate at slightly different depths but also with different orientations. The face-on position gives the higher result due to its orientation and its slightly reduced depth in the phantom.

\section{DISCUSSION AND CONCLUSIONS}

We have shown that TLD measures are a sensitive way to probe dose at different depths in small-animal phantoms. The techniques described here were sensitive enough to pick up variations due to stationary beam-on time, different depths, and different orientations. The TLD measurements were validated by ionization chamber measurements that gave a similar but, as expected, slightly higher dose rate. To save time, the dose rates measured here were for a single rotation over 53 seconds, acquiring 180 projection images rather than the usual 720 images used for a typical small animal study which would take approximately 200 seconds. The total exposure time included several seconds when the gantry was stationary before rotation and acquisition started. For the particular detector chip used, a mouse scan required 3 tiled images to capture the full projection image, this need will be removed when the tiled $2 \times 3$ MARS camera is used. The air kerma for a full 720 projection scan is estimated as $81.2 \mathrm{mGy}$ using the pencil chamber dose; this is a relatively high dose and could limit the number of scans that could be utilized in longitudinal studies.

As spectral CT develops there are several factors that will affect scanning protocols and thus total dose. In energy integrating detectors, high energy $\mathrm{x}$-rays are more heavily weighted even though they contribute less contrast information. This undesirable situation may be reversed for spectral CT where low energy x-rays could be given greater weight leading to higher contrast to noise ratios (CNR). Spectral detectors also avoid electronic and Swank noise which may also improve CNR or allow reduced dose. It is estimated that human CT utilizes in the order of $100 \times 10^{6}$ photons $/ \mathrm{s} / \mathrm{mm}^{2}$ for open beam, and 3 to $50 \times 10^{6}$ photons $/ \mathrm{s} / \mathrm{mm}^{2}$ for attenuated beam. This high count rate can result in pulse pile-up where the energy from coincident photons is incorrectly summed giving false energy information ${ }^{4}$. Novel scanner architecture and reconstruction processes may therefore be needed to provide accurate spectrometric data.

\section{REFERENCES}

[1] Anderson, N.G., Butler, A.P., Scott, N.J., Cook, N.J., Schleich, N., Firsching, M., Grasset, R., deRuiter, N., Campbell, M. and Butler, P.H., "Spectroscopic (multi-energy) CT distinguishes iodine and barium contrast material in mice," European Radiology 20(9), 2126-2134 (2010)

[2] Anderson, N. G. and Butler, A.P., "Clinical applications of spectral molecular imaging: potential and challenges," Contrast Media Mol. Imaging 9, 3-12 (2014)

[3] Yu, H., Wang, G., "Compressed sensing based interior tomography," Physics Medicine Biology 54, 2791-2805 (2009)

[4] Taguchi, K.,K Zhang, M., Frey, E.C., Wang, X., Iwanczyk, J.S., Nygard, E., Hartsough, N.E., Tsui, B.M.W., Barber, W.C., "Modelling the performance of a photon counting X-ray detector for CT: Energy response and pulse pileup effects," Medical Physics 38(2), 1089-1102 (2011) 
[5] Nik, S. J., Meyer, J. and Watts R., "Optimal material discrimination using spectral x-ray imaging," Phys. Med. Biol. 56, 5969-5983 (2011)

[6] Aamir R., Chernoglazov, A., Bateman, C.J., et al "MARS spectral molecular imaging of lamb tissue: data collection and image analysis, "Journal of Instrumentation 9, P02005 (2014)

[7] Walsh, M. F., Opie, A. M. T., Ronaldson, J. P., Doesburg, R. M. N., Nik, S. J., Mohr, J. L., Ballabriga, R., Butler, A. P. H., and Butler, P. H., "First CT using Medipix3 and the MARS-CT-3 spectral scanner," JINST 6(01), C01095 (2011)

[8] Ballabriga, R., Alozy, J., Blaj, G., Campbell, M., Fiederle, M., Frojdh, E., Heijne, E. H. M., Llopart, X., Pichotka, M., Procz, S., Tlustos, L. \& Wong, W., "The Medipix3RX: a high resolution, zero dead-time pixel detector readout chip allowing spectroscopic imaging," JINST, (2013).

[9] China Quartz Technology, "LiF for Dosimetry, Data Sheet, GR-200," version 101103, (22 December 2014) www.csimc-freqcontrol.com/img/Spec/Radioactive\%20test\%20materials/LiF/GR-200.pdf

[10] Duggan, L., Hood, C., Warren-Forward, H., Haque, M., Kron, T.,"Variations is dose response with x-ray energy of LiF:Mg,Cu,P thermoluminescence dosimeters: implications for clinical dosimetry," Phys. Med. Biol. 49, 3831-3845 (2004)

[11] Thermo Scientific, "Harshaw TLD Model 5500 Readers," (22 December 2014). www.thermoscientific.com/en/product/harshaw-tld-model-5500-readers-1.html

[12] Poludniowski, G.G., Evans, P.M., "Calculation of X-ray spectraemerging from an x-ray tube. Part I. Electron penetration characteristics in X-ray targets," Med. Phys. 34, 2164-2174 (2007)

[13] Poludniowski, G.G., "Calculation of x-ray spectra emerging from an X-ray tube. Part II.X-ray production and filtration in X-ray targets,"Med. Phys. 34, 2175-2186 (2007)

[14] RaySafe, "RaySafe Xi Specifications," (24 December 2014). http://mediabank.raysafe.com/A/RaySafe+Media+Bank/1599?encoding=UTF-8 